\title{
THE REAL BRAUER GROUP OF A FINITE CW-COMPLEX ${ }^{1}$
}

\author{
ELISABETTA STRICKLAND
}

\begin{abstract}
In this paper a complete description in homological terms of the real Brauer group $\mathrm{Br}_{R}(X)$ of a finite $\mathrm{CW}$-complex $X$ is obtained, in analogy with Jean Pierre Serre's result on the complex Brauer group $\mathrm{Br}_{C}(X)$ and the author's one for the orthogonal case. Precisely it is shown that
\end{abstract}

$$
\mathrm{Br}_{R}(X) \cong H^{2}\left(X, Z_{2}\right) \oplus H^{0}\left(X, Z_{2}\right)
$$

Introduction. Let $X$ be a finite CW-complex and $F(X)$ the set of fibre bundles on $X$, with fibres in Azumaya algebras over the field of real numbers $R$, that is to say central simple algebras over $R$. This set can be made into a semigroup with respect to the tensor product. Let $G(X)$ be the Grothendieck group of $F(X)$ modulo the relations

$$
a+b=a \otimes b \quad \forall a, b \in F(X) .
$$

The real Brauer group $\operatorname{Br}_{R}(X)$ of the space $X$ is the quotient of $G(X)$ modulo the elements of the form $\operatorname{End}(E), E$ being a real vector bundle on $X$.

Jean-Pierre Serre has shown [1, IV, Theorem 1.6] that $\operatorname{Br}_{C}(X) \cong$ Tor $H^{3}(X, Z)$, this being the torsion subgroup of $H^{3}(X, Z)$.

This result gives a complete description of the complex Brauer group in homological terms.

In [2] this problem is studied in the orthogonal case over the complex field and connections are found between $\mathrm{Br}_{C}(X)$ and $\mathrm{Br}_{C} \mathrm{O}(X)$. In the general linear case over the complex field the fibres of the fibre bundles are in the algebra of matrices $M_{n}(C)$ and by Skolem-Noether [3], the automorphisms of the algebra $M_{n}(C)$ are all inner automorphisms, and it is a standard construction to see that a classifying space for the Grothendieck group, which in this case we call $K P(X)$, is given by the space

$$
\operatorname{BPGL}_{C}(\infty)=\text { ind } \lim \mathrm{BPGL}_{C}(n)
$$

where the limit is made with respect to the maps induced by the homomorphisms

$$
\mathrm{PGL}_{C}(n) \stackrel{\otimes I}{\rightarrow} \mathrm{PGL}_{C}(n k), \quad k \in Z^{+},
$$

$I$ being the unit matrix [4].

Received by the editors March 23, 1978.

AMS (MOS) subject classifications (1970). Primary 54E60, 55D99, $55 \mathrm{~F} 10$.

${ }^{1}$ This research was supported by a C.N.R. grant at Brandeis University, Waltham, Massachusetts. 
Consider the fibration

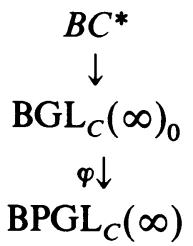

where $\mathrm{BGL}_{C}(\infty)_{0}=$ ind $\lim \mathrm{BGL}_{C}(n)$ is defined similarly to $\mathrm{BGPL}_{C}(\infty)$.

Note that by the standard construction of the localization for an $H$-space, it follows that $\mathrm{BGL}_{C}(\infty)_{0}$ is the localization of $\mathrm{BGL}_{C}(\infty)$, the classifying space for $\tilde{K}(X)[5,2.17]$.

In this way an element $x \in K P(X)$ belongs to the subgroup generated by the elements of type $\operatorname{End}(E)$, if and only if the corresponding element $\bar{x}$ in $\left[X, \mathrm{BPGL}_{C}(\infty)\right]$ lifts to an element of $\left[X, \mathrm{BGL}_{C}(\infty)_{0}\right]$.

$B C^{*}$ is the Eilenberg-Mac Lane space $K(Z, 2)$, and we obtain

$$
\begin{gathered}
{\left[X, \mathrm{BGL}_{C}(\infty)_{0}\right]=\tilde{K}(X) \otimes Q, \quad\left[X, \mathrm{BPGl}_{C}(\infty)\right]=K P(X),} \\
\operatorname{Br}_{C}(X)=K P(X) / \operatorname{Im} \varphi_{*} \tilde{K}(X) \otimes Q
\end{gathered}
$$

where $\varphi_{*}$ is the map induced by $\varphi$ among the sets of homotopy classes $\tilde{K}(X)$ and $K P(X)$.

One can give in quite a similar way to the general linear case over the complex field the definition of the orthogonal $\mathrm{Brauer}_{\text {group }} \mathrm{BrO}_{C}(X)$ of a finite $\mathrm{CW}$-complex $X$. This is obtained considering $\mathrm{BO}_{C}(\infty)_{0}$ instead of $\mathrm{BGL}_{C}(\infty)_{0}$ and $\mathrm{BPO}_{C}(\infty)$ instead of $\mathrm{BPGL}_{C}(\infty) . \mathrm{BPO}_{C}(\infty)$ is the classifying space of the functor $\mathrm{KPO}(-)$, which gives the Grothendieck group associated to the semigroup of fibre bundles in matrices with an orthogonal structure and $\mathrm{BO}_{C}(\infty)_{0}$ is the classifying space of $\tilde{\mathrm{K}} \mathrm{O} \otimes Q$.

Using homotopy theory, in [2] it is shown that

(a) $\mathrm{KPO}(X) \cong \bigoplus_{i=1}^{\infty} H^{4 i}(X, Q) \oplus H^{2}\left(X, Z_{2}\right)$,

(b) $\mathrm{BrO}_{C}(X) \cong H^{2}\left(X, Z_{2}\right)$.

Actually something more is pointed out: in view of the fact that if an element of $\mathrm{Br}_{C}(X)$ is representable by means of an orthogonal element, then it is an element of order 2, it is natural to investigate on the basis of Serre's result and (b) if an element of order 2 of $\mathrm{Br}_{C}(X)$ is representable by means of an element of $\mathrm{BrO}_{C}(X)$. An affirmative answer is given to the question as the map

(c) $\mathrm{BrO}_{C}(X) \stackrel{F^{\prime}}{\rightarrow} \mathrm{Br}_{C}(X)$

induced by the forgetful function because of (b) and Serre's isomorphism corresponds to the Bockstein homomorphism from $H^{2}\left(X, Z_{2}\right)$ to $H^{3}(X, Z)$, relative to the sequence $Z \rightarrow Z \rightarrow Z_{2}$. 
In other words, the diagram

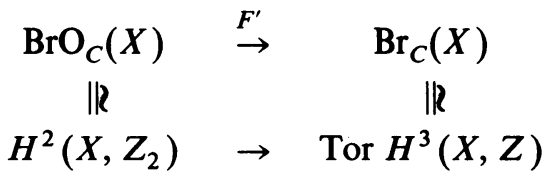

is commutative.

This gives an answer in the stable topologic case to one of a series of important problems connected with the elements of order 2 of the Brauer group, induced by a classical result due to A. Albert.

It is quite natural at this stage to give an answer to the following problem: if the base field is the one of real numbers $R$, find a homological description similar to the above mentioned ones, for the Brauer group of $X$. Of course the fact that the fibre bundles are now taken with fibres in central simple algebras over the field $R$ demands a more general argument than the one used in Serre's work or in [2].

But the complex orthogonal case will forward a part of the real Brauer group of $X$, as we shall prove that $\operatorname{Br}(X)$ splits into the following direct summand

$$
\mathrm{Br}_{R}(X) \cong H^{2}\left(X, Z_{2}\right) \oplus H^{0}\left(X, Z_{2}\right) .
$$

The main tools for achieving such result will be the knowledge of the Brauer group of the real numbers, $\operatorname{Br}(R)$, which has been proved to be isomorphic to $Z_{2}[1, \mathrm{p} .94]$, the homotopic equivalence of the general linear group over the real field, $\mathrm{Gl}_{R}(n)$, and the orthogonal group over the complex field $\mathrm{O}_{C}(n)$, and the properties of $\mathrm{Br}$ as a contravariant functor.

1. Some major observations. Let $\mathrm{Gl}_{R}(n)$ be the general linear group and $\mathrm{O}_{R}(n)$ the orthogonal group over $R$.

The natural inclusion of $\mathrm{O}_{R}(n)$ in the group $\mathrm{Gl}_{R}(n)$ induces homotopy equivalence between the corresponding classifying spaces.

This can be understood in the following way. The fibration

$$
\begin{gathered}
\mathrm{O}_{R}(n) \\
\downarrow \\
\mathrm{Gl}_{R}(n) \\
\downarrow \\
\mathrm{Gl}_{R}(n) / \mathrm{O}_{R}(n)
\end{gathered}
$$

leads to the following exact homotopy sequence

$$
0 \rightarrow \pi_{i}\left(\mathrm{O}_{R}(n)\right) \rightarrow \pi_{i}\left(\mathrm{Gl}_{R}(n)\right) \rightarrow \pi_{i-1}\left(\mathrm{Gl}_{R}(n) / \mathrm{O}_{R}(n)\right) \rightarrow \pi_{i-1}\left(\mathrm{O}_{R}(n)\right) \rightarrow
$$

which guarantees the group isomorphism

$$
\pi_{i}\left(\mathrm{O}_{R}(n)\right) \cong \pi_{i}\left(\mathrm{Gl}_{R}(n)\right) \quad \forall i
$$


as

$$
\pi_{i-1}\left(\mathrm{Gl}_{R}(n) / \mathrm{O}_{R}(n)\right)=0 .
$$

As a matter of fact, the space $\mathrm{Gl}_{R}(n) / \mathrm{O}_{R}(n)$ is contractible: its elements are positive symmetric matrices, so contractibility can be easily proved by using the Gram-Schmidt orthogonalization process. So, by a well-known theorem [5, Chapter 7, §6, p. 404], $\mathrm{O}_{R}(n)$ and $\mathrm{Gl}_{R}(n)$ are homotopically equivalent.

Similarly there is a natural inclusion as groups of $O_{R}(n)$ in $O_{C}(n)$, the last being the group of orthogonal $n \times n$ matrices over the complex field, and the fibration

$$
\begin{gathered}
\mathrm{O}_{R}(n) \\
\downarrow \\
\mathrm{O}_{C}(n) \\
\downarrow \\
\mathrm{O}_{C}(n) / \mathrm{O}_{R}(n)
\end{gathered}
$$

gives an exact homotopy sequence similar to (1.2), with

$$
\pi_{i}\left(\mathrm{O}_{R}(n)\right) \cong \pi_{i}\left(\mathrm{O}_{C}(n)\right)
$$

as $\mathrm{O}_{C}(n) / \mathrm{O}_{R}(n)$ is contractible too, being formed of matrices relative to positive hermitian forms, so Gram-Schmidt orthogonalization process can be applied here too.

Therefore we get that $\mathrm{O}_{C}(n)$ and $\mathrm{Gl}_{R}(n)$ are homotopically equivalent. But then it is known that the homotopy equivalence between the groups yields, by the Five Lemma, to the homotopy equivalence of the corresponding classifying spaces $\mathrm{BO}_{C}(n)$ and $\mathrm{BGL}_{R}(n)$.

As a matter of fact we get

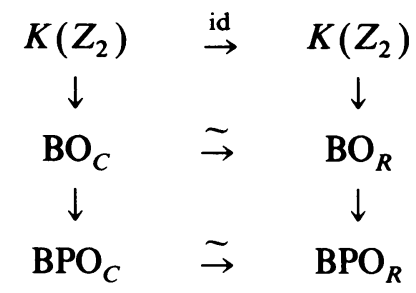

and

$$
\begin{array}{ccc}
K\left(Z_{2}, 1\right) & \stackrel{\sim}{ } & K\left(R^{*}, 1\right) \\
\downarrow & & \downarrow \\
\mathrm{BO}_{R} & \stackrel{\sim}{ } & \mathrm{BGL}_{R} \\
\downarrow & & \downarrow \\
\mathrm{BPO}_{R} & \stackrel{\sim}{\rightarrow} & \mathrm{BPGL}_{R}
\end{array}
$$

Diagrams (1.3) and (1.4) ensure, according to the construction of the Brauer group recalled in the introduction, which can readily be seen to 
respect the homotopic equivalence of $\mathrm{BO}_{C}(n)$ and $\mathrm{BGL}_{R}(n)$, that the part of the real Brauer group which comes from fibre bundles with fibres in matrix algebras, is the same as the orthogonal complex Brauer group, that is to say $H^{2}\left(X, Z_{2}\right)$.

The above argument will be of great help in $\$ 2$.

One more important tool that we need to accomplish our main purpose is forwarded by the following.

Proposition 1.5. Let $R$ be the field of real numbers. Then

$$
\operatorname{Br}(R) \cong Z / 2 Z \text {. }
$$

This result is pointed out by A. Grothendieck in the third of his well-known disquisitions on the Brauer group [1, Corollaire 2.3, p. 95] and can be readily understood using a theorem of Frobenius [7, $\$ \$ 11$ and 2, Theorem 2] which states that if $K$ is a maximal ordered division ring and $D$ a noncommutative division ring of $K$, of finite rank over $K$, and containing $K$ in its center, then there is a $K$-isomorphism from $K$ to the division ring of quaternions over $K$ corresponding to the couple $(-1,-1)$.

Proposition 1.5, tells us that if $W=\{x\}$ is a topological space reduced to a single point, $\operatorname{Br}_{R}(W) \cong Z_{2}$ and, if $\tilde{W}=\left\{\bigcup_{i \in I} x_{i}\right\}$, then

$$
\operatorname{Br}_{R}(\tilde{W}) \cong \bigoplus_{i \in I} Z_{2}^{i}
$$

2. The homological structure of $\operatorname{Br}_{R}(X)$. We are now ready to prove the following

2.1. TheOREM. Let $X$ be a finite $C W$-complex and $\operatorname{Br}_{R}(X)$ the real Brauer group of $X$. Then

$$
\mathrm{Br}_{R}(X)=H^{2}\left(X, Z_{2}\right) \oplus H^{0}\left(X, Z_{2}\right) .
$$

Proof. Let $X=\cup_{i \in I} X_{i}$, where $X_{i}$ are the connected components of the finite $C W$-complex $X$. Take in $X$ points $x_{1}, x_{2}, \ldots$ in such a way that $x_{i} \in X_{i}$, and consider the topological space $\left\{\cup_{i \in I} x_{i}\right\}$.

Consider the maps $\pi_{i}$ and $\varphi_{i}$ defined in the following way

$$
\left\{\bigcup_{i \in I} x_{i}\right\} \stackrel{\pi_{i}}{\rightarrow} X \stackrel{\varphi_{i}}{\rightarrow}\left\{\bigcup_{i \in I} x_{i}\right\} \quad \forall i \in I
$$

such that

$$
\pi_{i}\left(x_{i}\right)=x_{i} \text { and } \varphi_{i}(x)=x_{i} \quad \text { if } x \in X_{i}, \forall x \in X .
$$

Obviously $\varphi_{i} \circ \pi_{i}$ is the identity map of $\left\{\bigcup_{i \in I} x_{i}\right\}$.

So, as the Brauer group is a contravariant functor, we get, passing to the Brauer group of the spaces in (2.2),

$$
\mathrm{Br}_{R}\left(\left\{\bigcup_{i \in I} x_{i}\right\}\right) \stackrel{\varphi_{i}^{*}}{\rightarrow} \mathrm{Br}_{R}(X) \stackrel{\pi_{i}^{*}}{\rightarrow} \mathrm{Br}_{R}\left(\left\{\bigcup_{i \in I} x_{i}\right\}\right)
$$


and, as

$$
\operatorname{Br}_{R}\left(\left\{\bigcup_{i \in I} x_{i}\right\}\right) \cong \bigoplus_{i \in I} Z_{2}^{i} \cong H^{0}\left(\left\{\bigcup_{i \in I} x_{i}\right\}, Z_{2}\right)
$$

(2.3) becomes

$$
\bigoplus_{i \in I} Z_{2}^{i} \stackrel{\varphi_{i}^{*}}{\rightarrow} \mathrm{Br}_{R}(X) \stackrel{\pi_{i}^{*}}{\rightarrow} \bigoplus_{i \in I} Z_{2}^{i}
$$

the composition of $\pi_{i}^{*}$ and $\varphi_{i}^{*}, \pi_{i}^{*} \circ \varphi_{i}^{*}$ being the identity.

So we get a canonical splitting and the isomorphism

$$
\mathrm{Br}_{R}(X) \cong \operatorname{Ker} \pi_{i}^{*} \oplus\left(\bigoplus_{i \in I} Z_{2}^{i}\right) \cong \operatorname{Ker} \pi_{i}^{*} \oplus H^{0}\left(X, Z_{2}\right)
$$

Let us now detect $\operatorname{Ker} \pi_{i}^{*}$.

Let

$$
\begin{aligned}
& E \\
& \downarrow \\
& X
\end{aligned}
$$

be a fibre bundle on $X$, with fibres in central simple algebras and let us suppose that the class $[E] \in \operatorname{Br}_{R}(X)$ is such that $[E] \in \operatorname{Ker} \pi_{i}^{*}$. This implies that for any point $x_{i} \in X_{i}$, the fibre $E_{x_{i}}$ is a matrix algebra.

As a matter of fact (we need to prove this only for points in every connected component $X_{i}$ of $X$, as a fibre remains the same for each of these components), the class of $E_{x_{i}}$, with $x_{i} \in X_{i}$, in the Brauer group, [ $E_{x_{i}}$ ], is equal to 0 for every $i$, as the Brauer group of a point is the same as the Brauer group of the real numbers $R$.

So the fibre $E_{x_{i}}$ is a matrix algebra. But if the elements in Ker $\pi_{i}^{*}$ have representations which are matrix algebras, $\operatorname{Ker} \pi_{i}^{*} \cong H^{2}\left(X, Z_{2}\right)$, according to the argument pointed out in $\S 1$.

ACKNOWLEDGMENT. The author wishes to thank the advisor of this research, Professor Claudio Procesi.

\section{REFERENCES}

1. A. Grothendieck, Dix exposès sur la cohomologie des schemas, North-Holland, Amsterdam, 1968.

2. E. Strickland, On the orthogonal Brauer group, Atti Accad. Naz. Lincei Rend. Cl. Sci. Fis. Mat. Natur. (7) 62 (1977).

3. N. Bourbaki, Algèbre, Hermann, Paris, 1950-1958, Chapitres 4-6.

4. E. H. Spanier, Algebraic topology, McGraw-Hill, New York, 1966.

5. D. Sullivan, Geometric topology, Lecture notes, M.I.T., Cambridge, Mass., June 1970.

6. D. Husemoller, Fibre bundles, McGraw-Hill, New York, 1968.

7. N. Bourbaki, Modules et anneaux semisimples, Actualités Sci. Indust. no. 1261, Hermann, Paris, 1958.

8. G. Azumaya, On maximally central algebras, Nagoya Math. J. 2 (1951), 119-150.

istituto Matematico “G. Castelnuovo”, Università di Roma, Citta Universitaria, 00100 ROME, ITALY 